
allemande

49-1 | 2017

Berlin 1957-1994

\title{
La réforme du droit des sociétés luxembourgeois
}

chronique juridique

\section{Sandie Calme}

\section{(2) OpenEdition}

Journals

Édition électronique

URL : https://journals.openedition.org/allemagne/544

DOI : $10.4000 / a l l e m a g n e .544$

ISSN : 2605-7913

Éditeur

Société d'études allemandes

Édition imprimée

Date de publication : 16 juin 2017

Pagination : 243-247

ISSN : 0035-0974

Référence électronique

Sandie Calme, "La réforme du droit des sociétés luxembourgeois ", Revue d'Allemagne et des pays de langue allemande [En ligne], 49-1 | 2017, mis en ligne le 16 juin 2017, consulté le 01 juin 2022. URL : http://journals.openedition.org/allemagne/544 ; DOI : https://doi.org/10.4000/allemagne.544 


\section{Chronique juridique}

\section{La réforme du droit des sociétés luxembourgeois}

\section{- Sandie Calme*}

Le droit des sociétés luxembourgeois a fait l'objet d'une refonte récente qui conduit à la restructuration des sociétés relevant du droit luxembourgeois. Cette restructuration relève aussi de règlementations transitoires pour les sociétés de droit luxembourgeois préexistantes.

Ainsi, des dispositions transitoires prescrivent le passage progressif au nouveau régime pour une période de vingt-quatre mois, de sorte que les sociétés préexistantes qui ne modifient pas leurs statuts relèvent de l'ancien régime jusqu'au 23 août 2018 et de façon à ce que les dispositions de la réforme s'appliquent impérativement dès le 24 août 2018, toute disposition contraire étant réputée non écrite.

Désormais, l'article 2 de la loi sur les sociétés commerciales reconnaît comme "sociétés commerciales dotées de la personnalité juridique» les structures suivantes: la société en nom collectif, la société en commandite simple, la société anonyme et son corollaire la société par actions simplifiée, la société en commandite par actions, la société à responsabilité limitée, qui peut être simplifiée, la société coopérative et la société européenne.

La société en nom collectif est celle dans laquelle tous les associés sont indéfiniment et solidairement tenus de tous les engagements de la société (article 14 de la loi sur les sociétés commerciales). La société en commandite simple est celle que contractent, pour une durée limitée ou illimitée, un ou plusieurs associés indéfiniment et solidairement responsables des engagements sociaux, avec un ou plusieurs associés commanditaires qui n'engagent qu’une mise déterminée, constitutive de parts d'intérêts, représentées ou non par des titres conformément aux modalités prévues dans le contrat social (article 16 alinéa $1^{\text {er }}$ de la loi sur les sociétés commerciales). La société

* Docteur en droit, LL.M. (Francfort-sur-le-Main, Allemagne), avocate au barreau de Paris. 
en commandite spéciale se caractérise en ce qu'elle est dépourvue de la personnalité morale. La société anonyme est celle dont le capital est divisé en actions et qui est constituée par une ou plusieurs personnes qui n’engagent qu'une mise déterminée (article 23 alinéa $1^{\text {er }} a b$ initio de la loi sur les sociétés commerciales). La société en commandite par actions est celle que contractent, pour une durée limitée ou illimitée, un ou plusieurs actionnaires, indéfiniment et solidairement responsables des engagements sociaux, avec un ou plusieurs actionnaires qui n'engagent qu'une mise déterminée (article 102 de la loi sur les sociétés commerciales). Les sociétés à responsabilité limitée sont celles où des associés en nombre limité n'engagent qu'une mise déterminée, et dont les parts sociales représentées exclusivement par des titres non négociables ne peuvent être cédées que selon les prescriptions spéciales propres au droit luxembourgeois (article $179 a b$ initio de la loi sur les sociétés commerciales). À responsabilité limitée ou illimitée, les sociétés coopératives se composent d'associés dont le nombre et les apports sont variables et dont les parts sont incessibles à des tiers (article 113 de la loi sur les sociétés commerciales). La société européenne est issue du droit de l'Union Européenne.

Le vocable de société concerne également les sociétés momentanées et les sociétés en participation, autrefois désignées par le terme d'association, dépourvues de la personnalité morale. Les premières traitent une ou plusieurs opérations de commerce déterminées (article 138 de la loi sur les sociétés commerciales) et les secondes sont celles par lesquelles une ou plusieurs personnes s'intéressent dans des opérations qu'une ou plusieurs autres personnes gèrent en leur propre nom (article 139 de la loi sur les sociétés commerciales). Si leur consistance doit être prouvée, leurs dirigeants sont solidairement responsables envers les tiers avec qui ils ont traité.

Le Code civil luxembourgeois spécifie le droit commun des sociétés, qui s’applique également aux sociétés dont l'objet est civil, par définition non commerciales.

Désormais, d'après l'article 4 de la loi du 10 août 1915 modifié, les sociétés en nom collectif, les sociétés en commandite simple, les sociétés coopératives, les sociétés civiles, les sociétés en commandite spéciale et les sociétés à responsabilité limitée simplifiées sont, à peine de nullité, formées par des actes spéciaux notariés, mais peuvent l'être également par des actes spéciaux sous signatures privées. Cette possibilité de création par actes spéciaux sous signatures privées n'est pas ouverte aux autres formes sociales de droit luxembourgeois.

La réforme est inscrite dans la loi du 10 août 2016 portant modernisation de la loi du 10 août 1915 sur les sociétés commerciales entrée en vigueur le 23 août 2016 ainsi que dans la loi du 23 juillet 2016 instituant la société à responsabilité limitée («SARL.-S.») entrée en vigueur à compter du 16 janvier 2017.

Notoirement, le nouveau droit des sociétés luxembourgeois modernise l'organisation des sociétés anonymes et des sociétés à responsabilité limitée, met en place les sociétés par actions simplifiées et dispose des modalités de changement de forme sociale. 


\section{Réforme de la SARL}

\section{A. La SARL classique}

Les sociétés à responsabilité limitée, qui peuvent être unipersonnelles, sont celles où des associés en nombre limité n'engagent qu'une mise déterminée, et dont les parts sociales représentées exclusivement par des titres non négociables ne peuvent être cédées que conformément à un régime spécifique (article 179 de la loi sur les sociétés commerciales).

Elles ne peuvent être des sociétés d'assurances, de capitalisation et d'épargne (article 180 de la loi sur les sociétés commerciales).

Ainsi, pour ce qui concerne les SARL, le capital social minimum est désormais fixé à 12000 euros (article 182 de la loi sur les sociétés commerciales), le seuil de quarante actionnaires maximum passe à un maximum de cent actionnaires (article 181 de la loi sur les sociétés commerciales), les apports en industrie (article 183 in fine de la loi sur les sociétés commerciales) sont admis, donnant lieu à des parts sociales incessibles et intransmissibles et impliquant la contribution aux pertes, et permettent de participer au partage des bénéfices sans être inclus dans le capital social (article 183 in fine de la loi sur les sociétés commerciales), les SARL peuvent émettre des emprunts obligataires, des parts bénéficiaires et des parts rachetables. En outre, la cession de parts sociales à un tiers est assouplie par les options suivantes:

- les statuts de la SARL peuvent permettre l'agrément des associés sur la base de la moitié du capital social au lieu des trois quarts;

- au cas où le transfert n'est pas approuvé, les actionnaires restants sont autorisés à proposer des alternatives dans un délai de trois à six mois et au-delà de ce délai, l'actionnaire sortant concerné est en droit de transférer ses parts sociales au tiers initialement envisagé.

\section{B. La société à responsabilité limitée simplifiée ( $S A R L .-S$ )}

La forme sociale de société à responsabilité limitée simplifiée est une variante de la société à responsabilité limitée. Elle est basée sur le régime juridique de cette dernière, avec certains assouplissements. Elle ne regroupe que des personnes physiques qui ne peuvent faire partie que d'une SARL.-S., sauf si les parts sociales sont transmises à cause de mort. Le capital social initial d'une telle SARL doit être compris entre un et douze mille euros. Pour un capital de moins de douze mille euros, une réserve d'un vingtième des bénéfices nets est prévue. L'objet social de la société concerne les professions de la loi modifiée du 2 septembre 2011 réglementant l'accès aux professions d'artisan, de commerçant, d'industriel ainsi qu'à certaines professions commerciales.

\section{Réforme des sociétés anonymes}

Pour ce qui est des sociétés anonymes (ou «SA»), leur capital social minimal est fixé à 30000 euros, les actionnaires minoritaires voient leurs droits élargis, notamment en ce qui concerne le droit d'action contre les administrateurs de la SA, le personnel salarié peut gratuitement disposer d'actions, il est également possible d'émettre des obligations. Il y a également création d'une nouvelle forme sociale basée sur le modèle de la société anonyme, la société par actions simplifiée ( SAS»). 


\section{La société par actions simplifiée}

La mise en place de la forme sociale de société par actions simplifiée est conçue sur la base de la réforme française de 1994, en vue, notamment, d’une harmonie avec le droit des sociétés français. La société par actions simplifiée, relevant des articles 101-18 à 101-26 de la loi sur les sociétés commerciales, est une forme flexible de société anonyme. Elle ne saurait procéder à une émission publique d'actions. Elle est dirigée par un président qui la représente envers les tiers et envers la justice et auquel les statuts peuvent prévoir de substituer un ou plusieurs directeurs. Si le président ou un directeur est une personne morale, cette dernière intervient impérativement en la personne d'un représentant permanent qu'elle désigne. La responsabilité des dirigeants s'organise sur le modèle de la société anonyme et toute cession d'actions doit se conformer aux clauses statutaires.

\section{Possibilité de passage d'une forme sociale à une autre}

Le passage d'une forme sociale à une autre est strictement encadré.

La réforme du droit des sociétés luxembourgeois doit permettre le passage d'une forme sociale à une autre: il s'agit, le cas échéant, de choisir de passer à une nouvelle forme sociale en cohérence avec le droit des sociétés réformé.

Dès lors, les sociétés dont l'objet est civil pourront revêtir une forme de société commerciale: elles deviendront commerciales et devront se conformer aux lois et usages du commerce (loi du 10 août 2016).

Les sociétés civiles, quelle que soit l'époque de leur constitution, peuvent, si aucune disposition de leur contrat constitutif ne l'interdit, être transformées en sociétés à forme commerciale dotées de la personnalité juridique (article 2 alinéa $1^{\text {er }}$ de la loi de 1915 modifiée), par décision d'une assemblée générale spécialement convoquée à cet effet. Cette assemblée arrêtera les statuts de la société et sa décision ne sera valable que si elle obtient l'adhésion des titulaires de parts représentant les trois cinquièmes au moins des parts sociales (loi du 23 juillet 2016).

De même, les sociétés civiles pourront, quelle que soit l'époque de leur constitution, si aucune disposition de leur contrat constitutif ne l'interdit, être transformées en l'une des sociétés à forme commerciale, à l'exception de la société à responsabilité limitée simplifiée, par décision d'une assemblée générale spécialement convoquée à cet effet. Cette assemblée arrêtera les statuts de la société et sa décision ne sera valable que si elle obtient l'adhésion des titulaires de parts représentant les trois cinquièmes au moins des parts sociales (loi du 10 août 2016).

Un groupement européen d'intérêt économique pourra être transformé en une société dotée de la personnalité juridique, à l'exception de la société à responsabilité limitée simplifiée, en vertu de la loi du 23 juillet 2016, et vice-versa, une société dotée de la personnalité juridique pourra être transformée en groupement européen d'intérêt économique.

Chacune des sociétés dotées de la personnalité juridique, quelles que soient la nature primitive de son objet et l'époque de sa constitution, pourra, si aucune disposition de son contrat constitutif ne l'interdit, être transformée en une société de l'un des autres types prévus par ledit article 2 de la loi de 1915 modifiée, revêtue de la personnalité 
juridique ou non, ou en une société civile, à l'exception de la société européenne (SE) et de la société à responsabilité limitée simplifiée (loi du 23 juillet 2016).

Une société anonyme de droit luxembourgeois pourra se transformer en société européenne (SE) si elle a depuis au moins deux ans une société filiale relevant du droit d'un autre État membre de l'Espace Économique Européen (loi du 10 août 2016).

Les dispositions de la loi sur les sociétés commerciales relatives à la transformation sont également applicables à la transformation de personnes morales autres que des sociétés dans l'une des formes de sociétés dotées de la personnalité juridique en vertu de la présente loi de 1915 modifiée sur les sociétés commerciales, à l'exception de la société à responsabilité limitée simplifiée, dans la mesure où les lois particulières relatives à ces personnes morales le prévoient et dans le respect des dispositions spéciales de ces mêmes lois particulières (loi du 23 juillet 2016).

La société européenne (SE) ayant son siège statutaire au Grand-Duché de Luxembourg peut se transformer en société anonyme de droit luxembourgeois. La décision concernant la transformation ne peut être prise avant deux ans à partir de son immatriculation et avant que les deux premiers comptes annuels n'aient été approuvés (article 3 de la loi de 1915 modifiée sur les sociétés commerciales).

Il s'agit d'un ajustement justifié par la réforme: les transformations visées au présent article ne donnent lieu ni à dissolution ni à création d'une personnalité juridique nouvelle.

Toutefois, les droits des tiers, qui peuvent être des sociétés non luxembourgeoises, sont réservés, afin de garantir un certain degré de sécurité juridique.

En somme, la réforme du droit des sociétés luxembourgeois aspire à son attractivité, notamment pour les investissements internationaux. Elle implique une attention toute particulière en ce qui concerne les structures sociales transnationales dotées d'une implantation au Luxembourg. 